\title{
Performance analysis of GNSS/INS loosely coupled integration systems under GNSS signal blocking environment
}

\author{
Mengke Wang ${ }^{1, *}$, Peidong $\mathrm{Yu}^{1}$, and Yunzhi $\mathrm{Li}^{1}$ \\ ${ }^{1}$ College of Geodesy and Geomatics, Shandong University of Science and Technology, Qingdao 266000, Shandong, China
}

\begin{abstract}
Global Navigation Satellite System (GNSS) and Inertial Navigation System (INS) are the most widely used navigation systems at present. Aiming at the limitations of a single system application, this paper uses kalman filter to fuse the pose information provided by GNSS and INS, respectively. GNSS has the characteristics of being easily affected by the environment but with high absolute positioning accuracy. INS has the characteristics of high sampling frequency and autonomous navigation, but the error accumulates with time. Combining the advantages of the two systems to achieve the purpose of obtaining higher-precision pose information. In addition, aiming at the problem that GNSS/INS integration cannot provide continuous, stable and reliable navigation solutions under the GNSS signal blocking environment, a smoothing post-processing algorithm for GNSS/INS integration is studied. Through experimental verification, this algorithm can effectively improve the pose accuracy under GNSS signal blocking environment.
\end{abstract}

\section{Introduction}

In order to meet the requirements of high-precision navigation and mobile mapping, multiple navigation systems integrated has become a popular development direction. Global Navigation Satellite System (GNSS) and Inertial Navigation Systems (INS) are the typical candidates for integrated navigation systems ${ }^{[1]}$. GNSS has high long-term absolute positioning accuracy. However, under harsh observation environments, such as densely built cities, GNSS signals are vulnerable to interference and occlusion. When the number of observation satellites is less than 4, the condition of GNSS positioning solution cannot be satisfied. At this point, GNSS can no longer provide users with accurate position and velocity information $^{[2]}$. As an autonomous navigation system, INS is not affected by the external environment and has high positioning accuracy in the short term. And its sampling rate is generally higher than $100 \mathrm{HZ}$. However, the errors of inertial components will accumulate. If INS works alone for a long time, it will make the position and attitude information unavailable ${ }^{[4]}$. By comparing the advantages and disadvantages of GNSS and INS, it is not difficult to find that they are highly complementary.

GNSS/INS integrated navigation system has obvious advantages, and it has extremely broad application prospects both in the military field and surveying and mapping field. Therefore, so many researchers have done a lot of research on it. The three most common integration strategies are: loosely coupled, tightly coupled and deeply coupled. Deeply coupled is a integration at the hardware level. This technology can effectively solve the contradiction between performance and bandwidth setting in the traditional receiver tracking loop design. However, due to its complex combination structure and high cost, it has not been widely applied ${ }^{[4]}$. Tightly coupled uses the original observation of GNSS and INS navigation into the same filter. However, the data processing of tightly coupled is relatively complex. And due to the large amount of calculation and low navigation stability, it is difficult to realize in engineering ${ }^{[5]}$. Loosely coupled use the position and velocity output by GNSS to measurement update, and the output of the filter is used to correct the INS system error. This integration scheme is relatively easy to implement ${ }^{[6]}$. However, loosely coupled has a fatal flaw. When the number of observation satellites is less than 4, the GNSS module cannot output position and velocity information. At this point, the integration system degenerates into INS navigation system. Aiming at the serious influence of GPS signal blocking on GPS/INS integrated navigation system accuracy, HE designed a fuzzy strong tracking extended kalman filtering algorithm ${ }^{[7]}$. LI adds observation information to describe the motion characteristics of the carrier in vehicle navigation, and adds motion condition constraints of the carrier when GNSS signal blocking. This method can effectively reduce INS error accumulation ${ }^{[8]}$. Postprocessing is also a method to solve the positioning error accumulation of integration system caused by GNSS signal out-of-lock. MIAO presented the concrete realization form of RTS algorithm and experimentally verified the performance of the smoothing algorithm ${ }^{[9]}$. Liu focused on the backward filtering model in the smoothing algorithm, and gave the specific form of the model ${ }^{[10]}$.

Therefore, this paper studies a GNSS/INS loosely coupled smoothing post-processing algorithm, and gives

\footnotetext{
* Corresponding author: 1147547157@qq.com
} 
the system model and measurement model based on kalman filter, so as to improve the navigation solution accuracy of the integration system when GNSS signal is out-of-lock.

\section{Inertial-Frame Navigation Equations}

INS navigation algorithm is based on the explicit inertial navigation equation. When initial pose and Velocity are given, navigation information can be obtained recursively. The inertial system navigation equation used in this paper is based on the local navigation frame.

a. Position Differential Equations

$$
\left.\begin{array}{c}
\dot{L}=\frac{v_{E}}{\left(R_{E}+h\right) \cos B} \\
\dot{B}=\frac{v_{N}}{R_{N}+h} \\
h=v_{U}
\end{array}\right\}
$$

Where, $\mathrm{L}, \mathrm{B}, \mathrm{h}$ respectively represent the geographical longitude, latitude and height of the carrier. $\mathrm{v}_{\mathrm{E}}, \mathrm{v}_{\mathrm{N}}, \mathrm{v}_{\mathrm{U}}$ respectively represent the velocity of body frame with respect to ECEF, resolved about the three axes of the local navigation frame, which are east, north and up $. \mathrm{R}_{\mathrm{N}}, \mathrm{R}_{\mathrm{E}}$ respectively represent the meridian radius of curvature and transverse radius of curvature.

b. Velocity Differential Equations

$$
\dot{\boldsymbol{v}}^{n}=\boldsymbol{f}_{i b}^{n}-\left(2 \boldsymbol{\omega}_{i e}^{n}+\boldsymbol{\omega}_{e n}^{n}\right) \times \boldsymbol{v}^{n}+\boldsymbol{g}^{n}
$$

Where, $\omega_{\mathrm{ie}}^{\mathrm{n}}$ is the Earth-rotation resolved into local navigation frame. $\omega_{\mathrm{en}}^{\mathrm{n}}$ is the angular rate of local navigation frame with respect to ECEF. $\mathrm{f}_{\mathrm{ib}}^{\mathrm{n}}$ is specific force resolved into local navigation frame. $\mathrm{g}^{\mathrm{n}}$ is the local gravity vector of the carrier's position.

c. Attitude Differential Equations

$$
\dot{\boldsymbol{C}}_{b}^{n}=\boldsymbol{C}_{b}^{n} \mathbf{\Omega}_{n b}^{b}
$$

Where, $\mathrm{C}_{\mathrm{b}}^{\mathrm{n}}$ is coordinate transformation matrix of body frame with respect to local navigation frame. $\Omega_{\mathrm{nb}}^{\mathrm{b}}$ is a skew matrix of angular rate of body frame with respect to local navigation frame.

The pose of inertial navigation system is solved by strapdown algorithm updating. First, the angular rate measured by gyroscope is used to integrate Equation (3) to obtain the attitude update result. Then, the acceleration measured by accelerometer is used to integrate Equation (2) to obtain the velocity update result. Finally, the position update result is obtained by integrating equation (1) with the updated velocity result.

\section{GNSS/INS loosely coupled}

Fig. 1 is the structural diagram of the loosely coupled in this paper. The accelerometer and gyroscope of INS output the current specific force and angular rate of the carrier respectively. Then, the position, velocity and attitude can be obtained by the strapdown algorithm updating. In the GNSS module, the observation of the base station and the rover station is used to carry out differential GNSS calculation to get the position and velocity. The difference of position and velocity between INS and GNSS is used as the input of kalman filter. And the relevant information is stored during the filtering process. Then the state error estimate of inertial navigation system is obtained by integration navigation algorithm. The error estimate is used to correct the initial values of INS. And the information stored in the whole filtering process is used to smooth. The GNSS/INS integration system adopts closed-loop feedback correction, so that the state error can be kept at the optimal value. Finally, the position, velocity and attitude of the carrier with high accuracy and stability can be obtained.

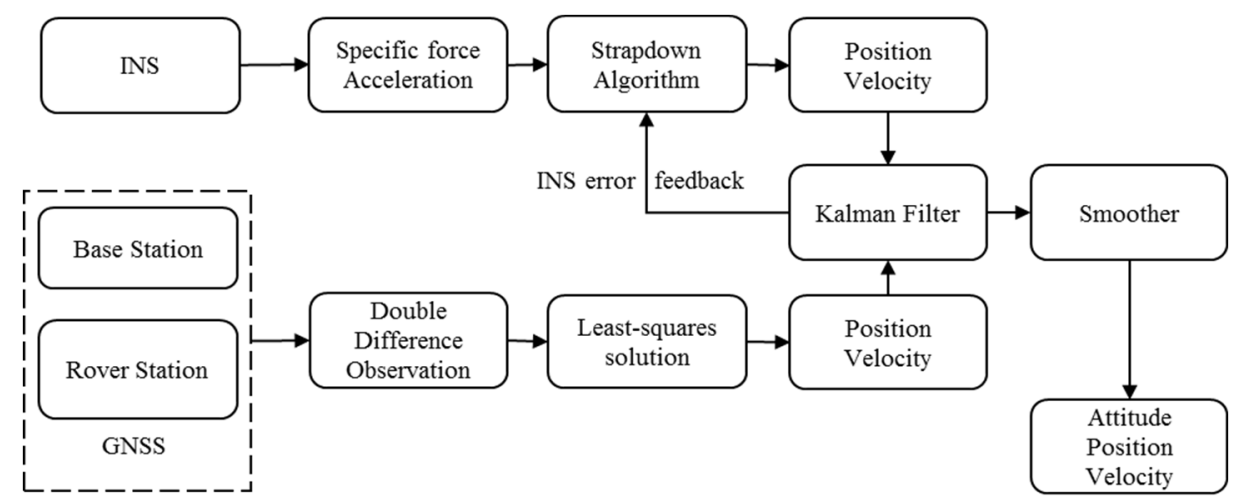

Fig. 1. Structure of the loosely coupled

\subsection{System mode}

GNSS/INS system model is based on the error equation of INS and the error model of inertial components. Error equation of inertial navigation system are:

$$
\left.\begin{array}{rl}
\dot{\boldsymbol{\varphi}}^{n}= & \delta \boldsymbol{\omega}_{i n}^{n}-\boldsymbol{\Omega}_{i n}^{n} \boldsymbol{\varphi}^{n}-\boldsymbol{C}_{b}^{n} \delta \boldsymbol{\omega}_{i b}^{b} \\
\delta \dot{\boldsymbol{v}}^{n}= & -\left(2 \delta \boldsymbol{\omega}_{i e}^{n}+\delta \boldsymbol{\omega}_{e n}^{n}\right) \times \boldsymbol{v}^{n} \\
- & \left(2 \boldsymbol{\omega}_{i e}^{n}+\boldsymbol{\omega}_{e n}^{n}\right) \times \delta \boldsymbol{v}^{n} \\
+ & {\left[\left(\boldsymbol{C}_{b}^{n} \boldsymbol{f}_{i b}^{b}\right) \times\right] \boldsymbol{\varphi}^{n}+\boldsymbol{C}_{b}^{n} \delta \boldsymbol{f}_{i b}^{b}} \\
\delta \dot{\boldsymbol{p}}^{n}=\boldsymbol{F}_{\boldsymbol{p} \boldsymbol{p}} \delta \boldsymbol{p}^{n}+\boldsymbol{F}_{\boldsymbol{p} \boldsymbol{v}} \delta \boldsymbol{v}^{n}
\end{array}\right\}
$$

Where, $\dot{\varphi}^{\mathrm{n}}, \delta \dot{\mathrm{v}}^{\mathrm{n}}, \delta \dot{\mathrm{p}}^{\mathrm{n}}$ respectively represent attitude error, velocity error and position error. $F_{p p}$ and $F_{p v}$ are coefficient matrix. Its specific form can be referred to references [11].

In addition, the error model of inertial components is:

$$
\left.\begin{array}{c}
\delta \boldsymbol{f}_{i b}^{b}=\delta \boldsymbol{b}_{g}+\boldsymbol{w}_{f} \\
\delta \boldsymbol{\omega}_{i b}^{b}=\delta \boldsymbol{b}_{a}+\boldsymbol{w}_{\omega}
\end{array}\right\}
$$

Where, $\delta b_{\mathrm{a}}$ and $\delta \mathrm{b}_{\mathrm{g}}$ respectively represent accelerometer bias and gyroscope bias. $\mathrm{w}_{\mathrm{f}}$ is the offset white noise of accelerometer. $\mathrm{w}_{\omega}$ is gyro drift white noise. 
The state vector is composed of attitude error, velocity error, position error, gyroscope measurement error and accelerometer measurement error, namely

$$
\mathrm{X}=\left[\left(\varphi^{\mathrm{n}}\right)^{\mathrm{T}},\left(\delta \mathrm{v}^{\mathrm{n}}\right)^{\mathrm{T}},\left(\delta \mathrm{p}^{\mathrm{n}}\right)^{\mathrm{T}},\left(\delta \mathrm{b}_{\mathrm{g}}\right)^{\mathrm{T}},\left(\delta \mathrm{b}_{\mathrm{a}}\right)^{\mathrm{T}}\right]^{\mathrm{T}}
$$

The state equation of GNSS/INS loosely coupled can be written as:

$$
\dot{\boldsymbol{X}}(t)=\boldsymbol{F}(t) \boldsymbol{X}(t)+\boldsymbol{G}(t) \boldsymbol{w}(t)
$$

Where, $F(t)$ is the system matrix. $G(t)$ is system noise distribution matrix. $w(t)$ is the system noise vector.

\subsection{Measurement model}

The observation of loosely coupled takes the difference between the output position and velocity of GNSS module and the output position and velocity of INS as the measurement information of kalman filtering. The measurement model of the system is as follows:

$$
\boldsymbol{Z}(t)=\left[\begin{array}{l}
r_{I N S}^{n}-r_{G N S S}^{n} \\
v_{I N S}^{n}-v_{G N S S}^{n}
\end{array}\right]
$$

Equation (7) is expressed as the measurement equation:

$$
\mathrm{Z}(\mathrm{t})=\mathrm{H}(\mathrm{t}) \mathrm{X}(\mathrm{t})+\mathrm{V}(\mathrm{t})
$$

Where, $\mathrm{Z}(\mathrm{t})$ is measurement vector. $\mathrm{H}(\mathrm{t})$ is measurement matrix. $V(t)$ is the white noise sources.

Discrete equations (6) and (8):

$$
\left.\boldsymbol{X}_{k}=\boldsymbol{\Phi}_{k, k-1} \boldsymbol{X}_{k-1}+\boldsymbol{\Gamma}_{k-1} \boldsymbol{w}_{k-1}\right\}
$$

Where, $X_{\mathrm{k}}$ is state vector at time $\mathrm{k} . \Phi_{\mathrm{k}, \mathrm{k}-1}$ is transition matrix from time $\mathrm{k}-1$ to time $\mathrm{k} . \Gamma_{\mathrm{k}-1}$ is the system noise distribution matrix from time $\mathrm{k}-1$ to time $\mathrm{k} . \mathrm{w}_{\mathrm{k}-1}$ is the system noise vector from time $\mathrm{k}-1$ to time $\mathrm{k} . \mathrm{Z}_{\mathrm{k}}$ is measurement vector at time $\mathrm{k} . \mathrm{H}_{\mathrm{k}}$ is measurement matrix at time $\mathrm{k} . \mathrm{V}_{\mathrm{k}}$ is the measurement noise vector at time $\mathrm{k}$.

\section{Kalman filtering and smoothing}

The kalman filtering process mainly consists of two parts: time update and measurement update. Its basic equation is:

$$
\left.\begin{array}{c}
\widehat{\mathrm{X}}_{\mathrm{f}, \mathrm{k} / \mathrm{k}-1}=\Phi_{\mathrm{k}, \mathrm{k}-1} \widehat{\mathrm{X}}_{\mathrm{f}, \mathrm{k}-1} \\
\mathrm{P}_{\mathrm{f}, \mathrm{k} / \mathrm{k}-1}=\Phi_{\mathrm{k}, \mathrm{k}-1} \mathrm{P}_{\mathrm{f}, \mathrm{k}-1} \Phi_{\mathrm{k}, \mathrm{k}-1}^{\mathrm{T}}+\Gamma_{\mathrm{k}-1} \mathrm{Q}_{\mathrm{k}-1} \Gamma_{\mathrm{k}-1}^{\mathrm{T}}
\end{array}\right\}
$$

Equation (10) describes the time update in kalman filter. In this process, the state estimator $\widehat{\mathrm{X}}_{\mathrm{f}, \mathrm{k}-1}$ and the covariance matrix $\mathrm{P}_{\mathrm{f}, \mathrm{k}-1}$ at time $\mathrm{k}-1$ predict the state vector $\widehat{X}_{\mathrm{f}, \mathrm{k} / \mathrm{k}-1}$ and error covariance matrix $\mathrm{P}_{\mathrm{f}, \mathrm{k} / \mathrm{k}-1}$ at time k. $Q_{k-1}$ is the system noise covariance matrix at time $\mathrm{k}-1$.

$$
\left.\begin{array}{c}
\boldsymbol{K}_{f, k}=\boldsymbol{P}_{f, k / k-1} \boldsymbol{H}_{k}^{T}\left(\boldsymbol{H}_{k} \boldsymbol{P}_{f, k / k-1} \boldsymbol{H}_{k}^{T}+\boldsymbol{R}_{k}\right)^{-1} \\
\widehat{\boldsymbol{X}}_{f, k}=\widehat{\boldsymbol{X}}_{f, k / k-1}+\boldsymbol{K}_{f, k}\left(\boldsymbol{Z}_{k}-\boldsymbol{H}_{k} \widehat{\boldsymbol{X}}_{f, k / k-1}\right) \\
\boldsymbol{P}_{f, k}=\left(\boldsymbol{I}-\boldsymbol{K}_{f, k} \boldsymbol{H}_{k}\right) \boldsymbol{P}_{f, k / k-1}\left(\boldsymbol{I}-\boldsymbol{K}_{f, k} \boldsymbol{H}_{k}\right)^{T} \\
+\boldsymbol{K}_{f, k} \boldsymbol{R}_{k} \boldsymbol{K}_{f, k}^{T}
\end{array}\right\}
$$

Equation (11) describes the measurement update in kalman filter, This process corrects the state vector according to the current time measurement vector, and then obtains the system state covariance matrix $P_{f, k}$ and system state estimate $\mathrm{X}_{\mathrm{f}, \mathrm{k}}$ at time $\mathrm{k} . \mathrm{K}_{\mathrm{f}, \mathrm{k}}$ and $\mathrm{R}_{\mathrm{k}}$ represent the kalman gain matrix and the Measurement noise covariance matrix at time $\mathrm{k}$ respectively.

In the above kalman filtering process, $\widehat{\mathrm{X}}_{\mathrm{f}, \mathrm{k}}, \Phi_{\mathrm{k}, \mathrm{k}-1}$,

$\mathrm{X}_{\mathrm{f}, \mathrm{k} / \mathrm{k}-1}, \mathrm{P}_{\mathrm{f}, \mathrm{k} / \mathrm{k}-1}$ at each moment is sequentially stored. The result of kalman filtering is taken as the initial value of reverse smoothing. Then the reverse smoothing algorithm is executed in reverse order:

$$
\left.\begin{array}{c}
\boldsymbol{A}_{k}=\boldsymbol{P}_{f, k} \boldsymbol{\Phi}_{k, k-1}^{T} \boldsymbol{P}_{f, k+1 / k}^{-1} \\
\widehat{\boldsymbol{X}}_{s, k}=\boldsymbol{X}_{f, k}+\boldsymbol{A}_{k}\left(\boldsymbol{X}_{s, k+1}-\widehat{\boldsymbol{X}}_{f, k+1 / k}\right) \\
\boldsymbol{P}_{s, k}=\boldsymbol{P}_{f, k}-\boldsymbol{A}_{k}\left(\boldsymbol{P}_{f, k+1 / k}-\boldsymbol{P}_{s, k+1}\right) \boldsymbol{A}_{k}^{T}
\end{array}\right\}
$$

Where, $A_{\mathrm{k}}$ is Smooth gain matrix, $\mathrm{X}_{\mathrm{s}, \mathrm{k}}$ is the state vector after smoothing, $\mathrm{P}_{\mathrm{s}, \mathrm{k}}$ is the covariance matrix after smoothing. In this paper, the error is taken as the filtering state vector, so after each filtering. The position, velocity, attitude information, gyroscope bias and accelerometer bias should be closed-loop corrected, and the state vector should be set to zero.

\section{Experimental analysis}

The experimental data used in this paper are measured by Vehicle mobile mapping system. The Vehicle mobile mapping system is equipped with NovAtel's SPan-LCI , GNSS receiver and other experimental equipment. The sampling rata of inertial data is $200 \mathrm{HZ}$, and that of GNSS data is $5 \mathrm{HZ}$.

Tab.1 Performance index of the SPAN-LCI

\begin{tabular}{ccc}
\hline Performance & Gyroscope & Accelerometer \\
\hline Bias stability & $<1.0^{\circ} / \mathrm{hr}$ & $<1.0 \mathrm{mg}$ \\
Random walk & $<0.05^{\circ} / \sqrt{\mathrm{hr}}$ & $50 \mu \mathrm{g} / \sqrt{\mathrm{Hz}}$ \\
Sampling rate & $200 \mathrm{HZ}$ & $200 \mathrm{HZ}$ \\
\hline
\end{tabular}

In order to study and analyze the performance of GNSS/INS loosely coupled smoothing algorithm under GNSS signal blocking environment, a section of experimental data with good reception of satellite signals was intercepted. And the artificial lockout of 60 s was performed on this section of data to simulate the lock-out environment. To verify the usefulness of the algorithm used in this article, navigation results processed by InertialExplorer8.60 (IE8.6) were used as reference values.

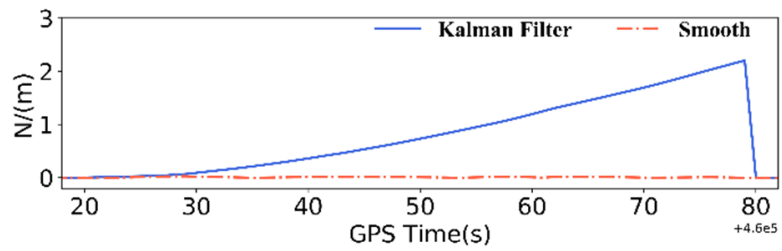

(a)

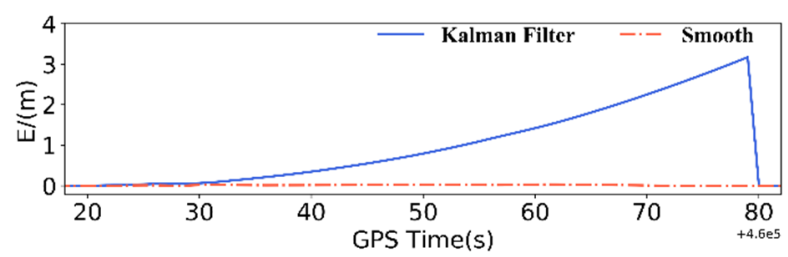

(b) 


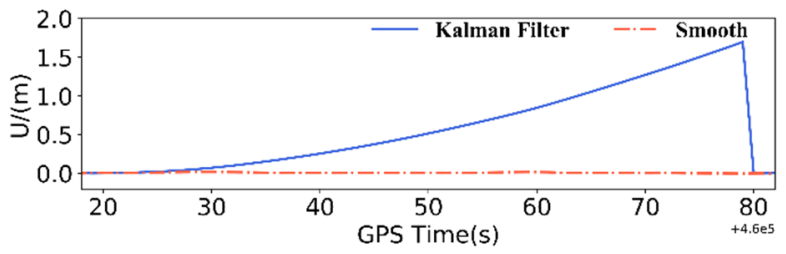

(c)

Fig. 2. Position error of GNSS/INS loosely coupled

It can be seen from Fig 1 that when GNSS signal is completely out of lock, GNSS module cannot provide accurate navigation solution for integration system. At this time, the inertial error accumulates with time, and the position error increases exponentially. After processing by the backward smoothing algorithm, the position error presents a smooth line, and the accuracy is higher. Combined with the position error statistical table (Table 2 ), when only forward Kalman filtering is used, the maximum error and RMS error in N, E and $\mathrm{U}$ all reach the level of meters, among which the maximum error in $\mathrm{E}$ direction reaches 3.175 meters and the root mean square (RMS) error reaches 1.422 meters. After backward smoothing, the maximum error of the three directions and the RMS error are all in centimeters. Thus, the backward smoothing algorithm greatly improves the precision of the combined navigation solution when the GNSS signal is out of lock.

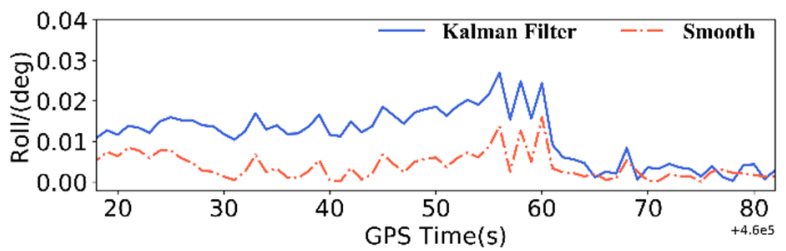

(a)

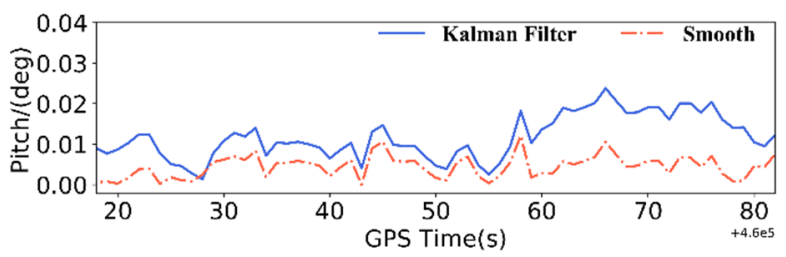

(b)

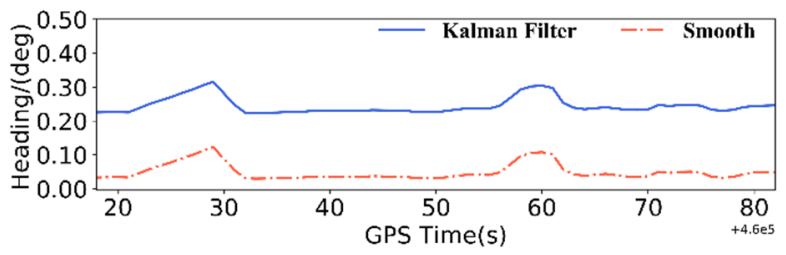

(c)

Fig. 3. Attitude error of GNSS/INS loosely coupled

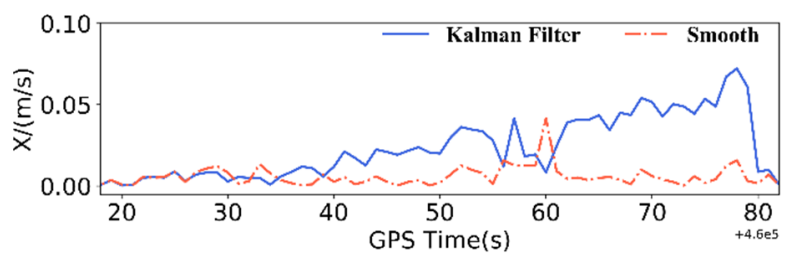

(a)

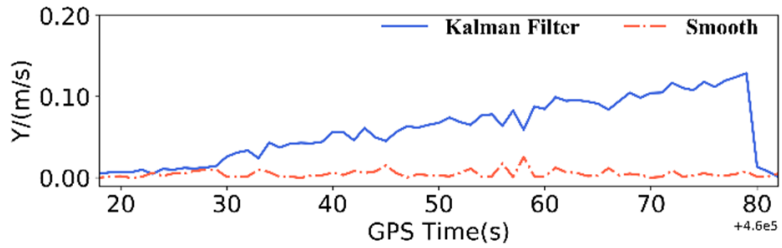

(b)

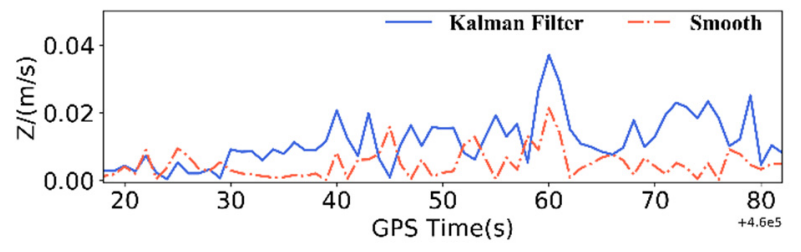

(c)

Fig. 4. Velocity error of GNSS/INS loosely coupled

Table.2 GNSS/INS combined algorithm position, velocity and attitude error statistics

\begin{tabular}{cccccc}
\hline & & \multicolumn{2}{c}{ Kalman Filter } & \multicolumn{2}{c}{ Smooth } \\
\cline { 3 - 6 } & & MAX & RMS & MAX & RMS \\
\hline \multirow{2}{*}{ Position } & $\mathrm{N} /(\mathrm{m})$ & 2.205 & 1.087 & 0.027 & 0.010 \\
error & $\mathrm{E} /(\mathrm{m})$ & 3.175 & 1.422 & 0.038 & 0.020 \\
& $\mathrm{U} /(\mathrm{m})$ & 1.699 & 0.807 & 0.017 & 0.011 \\
Velocity & $\mathrm{X}(\mathrm{m} / \mathrm{s})$ & 0.072 & 0.031 & 0.042 & 0.009 \\
error & $\mathrm{Y}(\mathrm{m} / \mathrm{s})$ & 0.129 & 0.073 & 0.026 & 0.007 \\
& $\mathrm{Z}(\mathrm{m} / \mathrm{s})$ & 0.037 & 0.014 & 0.010 & 0.007 \\
\multirow{2}{*}{ Attitude } & $\mathrm{Roll}\left({ }^{\circ}\right)$ & 0.027 & 0.014 & 0.016 & 0.005 \\
error & $\operatorname{Pitch}\left({ }^{\circ}\right)$ & 0.024 & 0.013 & 0.012 & 0.005 \\
& $\operatorname{Heading}\left({ }^{\circ}\right)$ & 0.316 & 0.247 & 0.123 & 0.006 \\
\hline
\end{tabular}

Combined with the velocity error statistical results in Table 2 and Fig 2, it can be seen that when the GNSS satellite is not available, the RMS error of the three directions of the integration system that only performs forward filtering is $3 \mathrm{~cm} / \mathrm{s}, 7 \mathrm{~cm} / \mathrm{s}$, and $1 \mathrm{~cm} / \mathrm{s}$. The maximum velocity error in three directions reaches decimeters per second. After the backward smoothing, the RMS error of the velocity in the three directions are limited to the level of millimeters per second. It can also be clearly seen in Fig 2 that smoothing improves the accuracy of velocity. It can be seen from Fig 3 that the attitude accuracy after smoothing has also been improved, among which the heading angle accuracy is the most obvious. Combined with the statistical results of the attitude errors in Table 2, the RMS error of the roll angle and pitch angle are $0.014^{\circ}$ and $0.013^{\circ}$, respectively. Due to the poor observability of the heading angle, the RMS error reaches $0.247^{\circ}$. After smoothing, the RMS error of the roll angle and pitch angle are both $0.005^{\circ}$, and the accuracy of the heading angle is also greatly improved.

\section{Conclusion}

In this paper, a GNSS/INS loosely coupled smoothing post-processing is studied. First, the GNSS solution and the INS solution are fused through the kalman filter, and the error of the inertial components is corrected by the state vector which output by the Kalman filter, and then the integration system is further processed by the smoothing post-processing algorithm. Finally, by 
artificially simulating the GNSS signal loss-of-lock environment, the performance analysis of the integration system post-processing algorithm is carried out. Experiments show that, in the case of GNSS satellite loss of lock, smooth post-processing algorithm as a bridge algorithm can also greatly improve positioning accuracy.

\section{Reference}

1. Li Z, Zhang L, Chen S, Yang C, Ma L. Overview and prospect of strapdown inertial/satellite ultra-tight integrated navigation technology . Systems engineering and electronics, 38(04),866-874,(2016).

2. Li, X., Zhang, X., Ren, X. Precise positioning with current multi-constellation Global Navigation Satellite Systems: GPS, GLONASS, Galileo and BeiDou. Sci Rep 5, 8328 (2015).

3. Xue L, Shen Y, Song L, Chen X. A review of foreign navigation technology development in 2019. Navigation and control, 19(02),1-9,(2020)

4. Niu X, Ban Y, Zhang J, Liu J. Research progress and prospect of GNSS/INS deep combination technology. Acta aerophenica sinica ,37(10),2895-2908. (2016)

5. Wang J, Liu D, Jiang W. Evaluation on loosely and tightly coupled GNSS/INS vehicle navigation system[C], ICEAA,(2017).

6. Qiu L, Yao Y, Zhu C. Comparison of the realization and positioning accuracy of GPS/INS loose combination and tight combination. Surveying and mapping geographic information ,38(03),17-19, (2013).

7. He K, Dong C, Wang Q. A navigation filtering algorithm considering GPS signal interruption . Journal of Beijing university of aeronautics and astronautics, 45(09),1874-1881,(2019).

8. Li Y, Yang Y, He H. Impact analysis of additional constraints on GNSS/INS integrated navigation results . Journal of wuhan university (information science edition), 42(09),1249-1255,(2017).

9. Miao Y. Research on data Processing Method of SINS/GPS Integrated Navigation. Information Engineering University,(2013).

10. Liu S, Sun F, Li H, Zhang L. Application of forward and backward smoothing algorithm in postprocessing of precise single point positioning /INS compact combination data. Chinese journal of inertial technology,23(01):85-91,(2015).

11. Liu S. Research and Implementation of GPS/INS Integrated Navigation Algorithm. Information Engineering University,(2012). 